\title{
Vitamin A, vitamin E, iron and zinc status in a cohort of HIV-infected mothers and their uninfected infants
}

\author{
Jacqueline Pontes Monteiro ${ }^{[1]}$, Maria Letícia Santos Cruz ${ }^{[2]}$, Marisa Márcia Mussi-Pinhata ${ }^{[1]}$, \\ Roberta Garcia Salomão $0^{[1]}$, Alceu Jordão Junior ${ }^{[3]}$, Laura Freimanis Hance ${ }^{[4]}$, \\ Jennifer Suzanne Read ${ }^{[5],[6]}$, José Henrique da Silva Pilotto[7],[8], Rachel Ann Cohen ${ }^{[4]}$, \\ Sonia Karolina Stoszek ${ }^{[4]}$ and George Kelly Siberry ${ }^{[5]}$
}

[1]. Departamento de Puericultura e Pediatria, Faculdade de Medicina de Ribeirão Preto, Universidade de São Paulo, Ribeirão Preto, SP, Brasil. [2]. Departamento de Doenças Infecciosas, Hospital Federal dos Servidores do Estado, Rio de Janeiro, RJ, Brasil. [3]. Departamento de Clínica Médica, Faculdade de Medicina de Ribeirão Preto, Universidade de São Paulo, São Paulo, SP, Brasil. [4]. Westat, Rockville, Maryland, USA. [5]. Maternal and Pediatric Infectious Disease Branch, National Institute of Child Health and Human Development, National Institute of Health, Bethesda, Maryland, USA. [6]. Division of Infectious Diseases, Department of Pediatrics, The George Washington University School of Medicine, Washington, District of Columbia, USA. [7]. Departamento de Doenças Infecciosas, Hospital Geral de Nova Iguaçu, Nova Iguaçu, RJ, Brasil. [8]. Laboratório de AIDS e Imunologia Molecular, Instituto Oswaldo Cruz, Fundação Oswaldo Cruz, Rio de Janeiro, RJ, Brasil.

\begin{abstract}
Introduction: We hypothesized that nutritional deficiency would be common in a cohort of postpartum, human immunodeficiency virus (HIV)-infected women and their infants. Methods: Weight and height, as well as blood concentrations of retinol, $\alpha$-tocopherol, ferritin, hemoglobin, and zinc, were measured in mothers after delivery and in their infants at birth and at 6-12 weeks and six months of age. Retinol and $\alpha$-tocopherol levels were quantified by high performance liquid chromatography, and zinc levels were measured by atomic absorption spectrophotometry. The maternal body mass index during pregnancy was adjusted for gestational age (adjBMI). Results: Among the 97 women 19.6\% were underweight. Laboratory abnormalities were most frequently observed for the hemoglobin $(46.4 \%)$, zinc $(41.1 \%)$, retinol $(12.5 \%)$ and ferritin $(6.5 \%)$ levels. Five percent of the women had mean corpuscular hemoglobin concentrations $<31 \mathrm{~g} / \mathrm{dL}$. The most common deficiency in the infants was $\alpha$-tocopherol (81\%) at birth; however, only $18.5 \%$ of infants had deficient levels at six months of age. Large percentages of infants had zinc (36.8\%) and retinol (29.5\%) deficiencies at birth; however, these percentages decreased to $17.5 \%$ and $18.5 \%$, respectively, by six months of age. No associations between infant micronutrient deficiencies and either the maternal adjBMI category or maternal micronutrient deficiencies were found. Conclusions: Micronutrient deficiencies were common in HIVinfected women and their infants. Micronutrient deficiencies were less prevalent in the infants at six months of age. Neither underweight women nor their infants at birth were at increased risk for micronutrient deficiencies.
\end{abstract}

Keywords: Micronutrients. HIV infection. Pregnancy. Infant. Nutrition. Cohort.

\section{INTRODUCTION}

Maternal malnutrition is associated with increased risks of infant morbidity and mortality ${ }^{1}$. Multiple micronutrient deficiencies may develop in pregnant women early during human immunodeficiency virus type 1 (HIV-1) infection ${ }^{2,3}$. Zinc deficiency, which is common in HIV-infected individuals, has been associated with higher mortality and viral load ${ }^{4,5}$. Zinc supplementation during pregnancy results in improved neonatal immune status, reduced early neonatal morbidity

Address to: Dra. Jacqueline Pontes Monteiro. Depto. Puericultura e Pediatria/ FMRP/USP. Av. Bandeirantes 3900, Campus USP, 14049-900 Ribeirão Preto, SP, Brasil.

Phone: 55 16 3602-2477; Fax: 5516 3602-2700

e-mail: jacque@fmrp.usp.br

Received 13 October 2014

Accepted 18 November 2014 and fewer infant infections ${ }^{6}$. Pregnancy increases the risk of vitamin A deficiency in mothers and newborns. Randomized trials of vitamin A supplementation in pregnant women found decreased mortality and a reduced prevalence and duration of infectious episodes in infants ${ }^{7}$. In a Brazilian study, maternal vitamin A deficiency was strongly associated with infant vitamin A deficiency and low birth weight ${ }^{8}$. Brazilian HIV-infected pregnant women may have low vitamin A levels even after supplementation'.

HIV infection and pregnancy are accompanied by oxidative stress. Lower levels of vitamin E may play a pathogenic role in the onset and development of acquired immunodeficiency syndrome (AIDS) and other infectious diseases ${ }^{10,11}$. Several studies have revealed lower vitamin E levels in HIV-infected children in Brazil and elsewhere ${ }^{12-14}$. Among healthy pregnant women, infant birth weight and length are associated with maternal serum concentrations of antioxidant vitamins ${ }^{15}$. Anemia is the most frequent hematologic abnormality found in HIV disease ${ }^{16}$. Standard prenatal care for HIV-infected pregnant 
women in Latin America includes iron and folate supplements to prevent anemia and neural tube defects.

We hypothesized that nutritional deficiency is frequent among HIV-infected mothers and their infants in Brazil. Our objectives were as follows: 1) to describe the nutritional status of HIV-infected women at delivery and of their infants at sites in Brazil based on anthropometric measurements, including the maternal adjusted for gestational age (adjBMI), and on plasma micronutrient concentrations; 2 ) to assess the correlation between maternal and infant micronutrient levels at delivery; and 3) to evaluate changes in the micronutrient status of infants from birth to six months of age.

\section{METHODS}

\section{The LILAC study}

HIV-infected pregnant women enrolled at three participating Brazilian sites in the Eunice Kennedy Shriver National Institute of Child Health and Human Development (NICHD), International Site Development Initiative (NISDI) and Longitudinal Study in Latin American Countries (LILAC) dynamic cohort were invited to participate in this nutritional LILAC sub-study. The design and conduct of the LILAC study has been described previously ${ }^{17}$.

\section{Nutritional Sub-study}

This nutritional sub-study enrolled LILAC participants at three study sites in Brazil. The protocol for this sub-study was approved by the Institutional Review Boards (IRBs) of the participating institutions. This sub-study required the collection of additional blood from mothers (within seven days of delivery) and infants (at birth and then at the 6-12-week and 6-month study visits) for the analysis of micronutrient levels (retinol, $\alpha$-tocopherol, ferritin, and zinc). The blood samples were stored at $-70^{\circ} \mathrm{C}$ in the dark until processing. Plasma retinol and $\alpha$-tocopherol concentrations were quantified by high performance liquid chromatography (HPLC) as previously described ${ }^{18}$. Serum zinc was determined by atomic absorption spectrophotometry using standard procedures ${ }^{19}$. Maternal C-reactive protein (CRP) levels were quantified to characterize inflammation. CRP and ferritin were measured in plasma samples using immunoassay technology (Immulite 1,000 Immunoassay System - Global Siemens Healthcare Headquarters - Siemens AG; Henkestrasse 127; D-91052 Erlangen - Germany).

The maternal body mass index (BMI) at the last study visit during pregnancy was adjusted for gestational age (adjBMI) using a computer algorithm provided by the Ministry of Health of Argentina ${ }^{20}$ and was used as a criteria for nutritional status classification. Maternal adjBMI values that were $<19.8$ were considered underweight, $\geq 26.1$ to $<29$ were considered overweight, and $\geq 29$ to $<50$ were classified as obese. Maternal and infant micronutrient status was defined at each time point as deficient vs. normal for each micronutrient measured. Deficiencies were defined as follows for each micronutrient: zinc level, $<64 \mathrm{mg} / \mathrm{dL}$ for infants and $<50 \mathrm{mg} / \mathrm{dL}$ for mothers ${ }^{21}$; retinol level, $<0.7 \mathrm{mmol} / \mathrm{L}$ for both infants and mothers ${ }^{22}$; $\alpha$-tocopherol level, $<12 \mathrm{mmol} / \mathrm{L}$ for infants and $<7 \mathrm{mmol} / \mathrm{L}$ for mothers ${ }^{23}$; and ferritin level, $<10 \mathrm{ng} / \mathrm{mL}$ for mothers, $<25 \mathrm{ng} / \mathrm{mL}$ for infants less than one month of age, $<200 \mathrm{ng} / \mathrm{mL}$ for infants one to two months of age, $<50 \mathrm{ng} / \mathrm{mL}$ for infants aged two months to less than six months, and $<7 \mathrm{ng} / \mathrm{mL}$ for ages six months to 15 years $^{24}$. The cut-off values for C-reactive protein (CRP) categories in pregnant women were as follows: low, $<1 \mathrm{mg} / \mathrm{dL}$; normal, 1-3mg/dL; high, $>3 \mathrm{mg} / \mathrm{dL}^{25}$. Infant CRP cut-off values were as follows: normal, $\leq 0.5 \mathrm{mg} / \mathrm{dL}$; high, $>0.5 \mathrm{mg} / \mathrm{dL}^{26}$. HIV disease was classified according to the Centers for Disease Control and Prevention (CDC) definitions ${ }^{27}$. A low mean corpuscular hemoglobin concentration (MCHC) level was defined as $<31 \mathrm{~g} /$ $\mathrm{dL}$ for pregnant women and for infants ${ }^{24}$. The cut-off values for hemoglobin in pregnant women were as follows: severe deficiency, $6.5-7.4 \mathrm{~g} / \mathrm{dL}$; low, $7.5-8.4 \mathrm{~g} / \mathrm{dL}$; mild deficiency, $8.5-10 \mathrm{~g} / \mathrm{dL}$; and normal, $>10 \mathrm{~g} / \mathrm{dL}^{28}$. The normal hemoglobin range for infants was adjusted for age: one to three days, 14.5-18.5g/ dL; two weeks, 13.4-16.6g/dL; one month, 10.7-13.9g/dL; two months, 9.4-11g/dL; and from two to six months, 11.1-12.6g/ $/ \mathrm{dL}^{28}$.

Maternal antiretroviral (ARV) regimens were categorized in order of regimen complexity as follows: 1) one or two nucleoside reverse transcriptase inhibitors (NRTIs), 2) two NRTIs and one non-nucleoside reverse transcriptase inhibitor (NNRTI), 3) two NRTIs and one protease inhibitor (PI), and 4) other. The reason for the use of ARVs during pregnancy was categorized as treatment if ARVs were used before pregnancy and/or continued after the 6-12-week study visit, or as prophylaxis if ARVs were initiated during pregnancy and use was discontinued by the 6-12-week visit.

\section{Statistical analysis}

Associations between maternal adjBMI values and the following characteristics of mothers and infants were examined: maternal age at enrollment, maternal years of formal education, number of persons in the household, mother's gainful employment outside the home, substance use during pregnancy, reasons for ARV use during pregnancy, maternal ARV regimen, maternal cluster of differentiation 4 (CD4) + T-lymphocyte (CD4) count, plasma Human immunodeficiency virus-ribonucleic acid (HIV-RNA) concentration [viral load(VL)], CDC clinical HIV disease classification ${ }^{27}$, gestational hypertension, gestational diabetes, preterm birth $(<37$ weeks of gestational age $)^{1}$, low infant birth weight $(<2,500 \mathrm{~g})^{1}$, infant ARV regimen and CD4+ T-lymphocyte count at birth. Associations between maternal adjBMI and maternal and infant micronutrient levels, which were categorized as deficient vs. normal, were examined. Additionally, associations between micronutrient levels in mothers and infants were examined. The maternal vitamin $\mathrm{A}$, vitamin $\mathrm{E}$, iron, and zinc levels were examined within seven days of delivery, and infant micronutrient status was examined at birth, 6-12 weeks, and six months of age. These associations were examined using Fisher's exact test or the Fisher-Freemen-Halton extension to Fisher's exact test. Significant bivariate results were further investigated on the basis of relative risks (RRs) and $95 \%$ confidence intervals ( $95 \%$ CIs) were obtained from log-binomial regression modeling. Linear regression and one-way analysis of variance (ANOVA) were used to examine associations between continuous maternal 
and infant nutritional variables at the study time points. All $\mathrm{p}$-values were two-sided. The $\mathrm{p}$-values $<0.05$ were considered statistically significant. Analyses were conducted using SAS version 9.3 statistical software (SAS Institute, Cary, NC, USA).

\section{RESULTS}

In total, 123 women that were enrolled in the LILAC protocol at the participating sites provided informed consent to participate in this sub-study, and blood samples were available for 97 mother-infant pairs. Thus, the study population was composed of 97 mother-infant pairs. All infants were HIVuninfected.

The characteristics of the 97 mother-infant pairs overall and according to maternal adjBMI at the last study visit during pregnancy are shown in Table 1. Based on the adjBMI measurements, $26(26.8 \%)$ of the mothers were classified as overweight/obese, $52(53.6 \%)$ were normal weight, and 19 (19.6\%) were underweight. The reason for using ARVs during pregnancy was significantly associated with adjBMI ( $p$-value $=0.03$ ); a higher proportion of overweight/obese women received ARVs for prophylaxis (as opposed to treatment) during

TABLE 1 - Characteristics of 97 mother-infant pairs by maternal adjBMI.

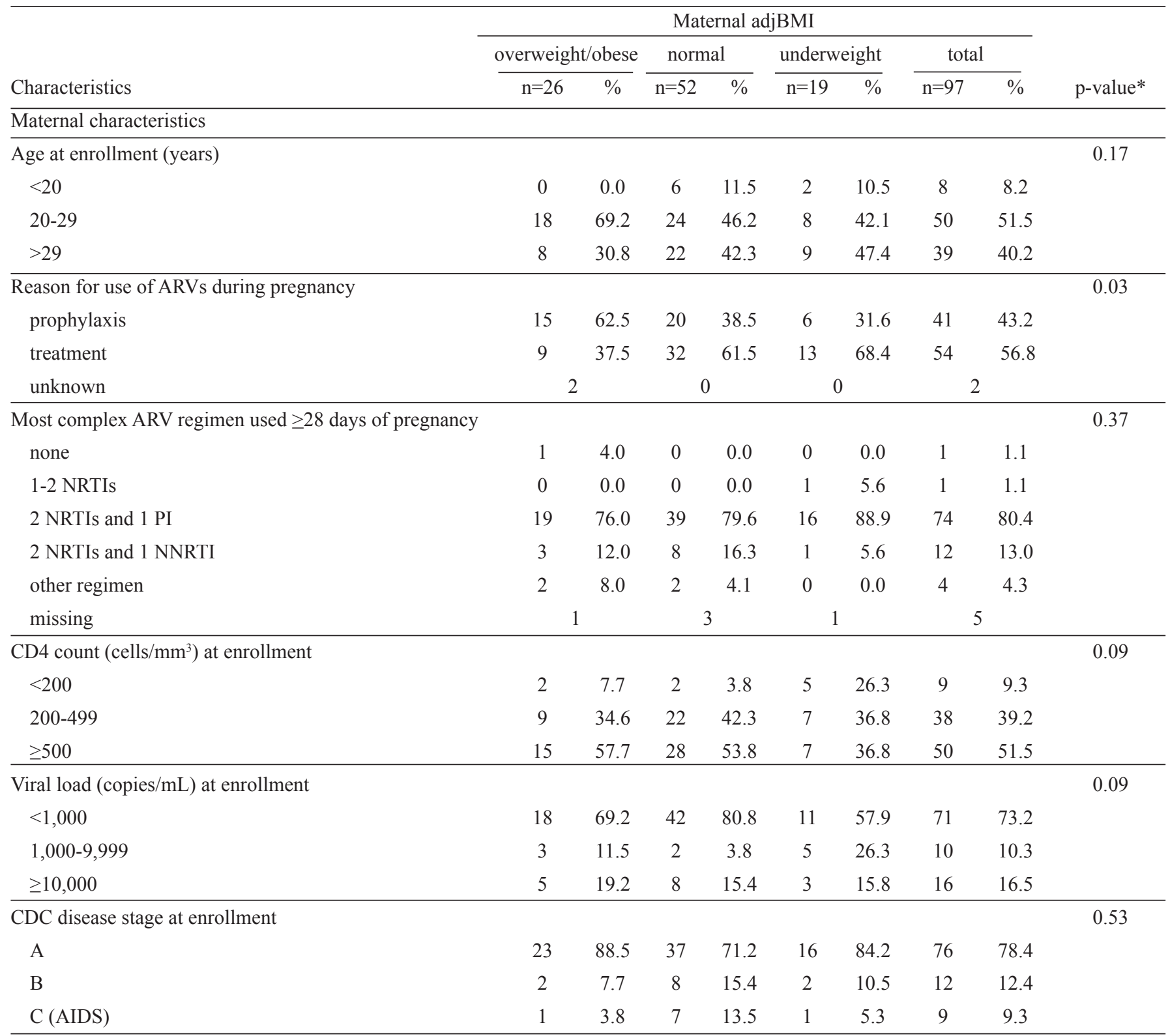

*Fisher's exact test. adjBMI: body mass index adjusted for gestational age; ARV: antiretroviral; NRTI: nucleoside reverse transcriptase inhibitors; PI: protease inhibitor; NNRTI: non-nucleoside reverse transcriptase inhibitor; CD4: cluster of differentiation 4; CDC: Centers for Disease Control and Prevention. 
pregnancy compared with normal weight or underweight women. No associations were observed between maternal adjBMI and years of education ( $p$-value $=0.45$ ), the number of persons in the household ( $\mathrm{p}$-value $=0.41$ ), gainful employment outside the home ( $\mathrm{p}$-value $=0.54)$, tobacco use during pregnancy $(\mathrm{p}$-value $=0.74)$, alcohol use during pregnancy $(\mathrm{p}$-value $=0.71)$, cocaine use during pregnancy $(\mathrm{p}$-value $=0.42)$ or marijuana use during pregnancy ( $\mathrm{p}$-value $=0.42$ ). Additionally, maternal adjBMI was not associated with preterm birth ( $p$-value $=0.77$ ), low birth weight ( $\mathrm{p}$-value $=0.28$ ) or infant CD4 count at birth ( $p$-value $=0.59)$. The median gestational age at the time of adjBMI assessment was 33 weeks (minimum, 25 weeks; maximum, 39 weeks). Underweight women were significantly more likely to have low MCHC after delivery than their normal and overweight counterparts ( $\mathrm{p}$-value $<0.01$; data not shown).

Nutritional and inflammatory marker data for the mothers and infants are summarized [mean, standard deviation (SD)], median, and range) in Table 2. The test results are categorized as normal versus deficient in Table 3. The most frequent maternal deficiencies were zinc $(41.1 \%)$, followed by retinol $(12.5 \%)$, ferritin $(6.5 \%)$ and $\alpha$-tocopherol (1\%) (Table 3). Additionally, $46.4 \%$ of the women had low hemoglobin levels, and the $\mathrm{MCHC}$ was $<31 \mathrm{~g} / \mathrm{dL}$ in $5.2 \%$ of the women. Approximately $54 \%$ of the women had high levels of CRP in their blood at hospital discharge following delivery. At birth, $81.1 \%$ of the infants were deficient in $\alpha$-tocopherol, with the proportion decreasing with age to only $18.5 \%$ by six months of age. High proportions of neonates also had deficient levels of zinc $(36.8 \%)$ and retinol $(29.5 \%)$ at birth, and these numbers fell to only $17.5 \%$ and $18.5 \%$, respectively, by six months of age. The proportion of infants with low levels of retinol increased to $41.1 \%$ by $6-12$ weeks of age before decreasing by six months of age. Although none of the infants had low ferritin levels at birth, a sharp increase was observed in the proportion of infants with low ferritin levels at 6-12 weeks of age $(58.7 \%)$ and at 6 months of age (25.8\%). Additionally, low hemoglobin levels were observed in $43.8 \%$ and $7.5 \%$ of the infants at $6-12$ weeks of age and at 6 months of age, respectively. All infants were formula-fed after birth.

When we compared the underweight adjBMI category with the normal adjBMI category, no association was found between maternal underweight adjBMI during pregnancy and maternal zinc $(\mathrm{RR}=1.2,95 \% \mathrm{CI}: 0.7,2.3)$, retinol $(\mathrm{RR}=0.7,95 \%$ CI: $0.2,2.9$ ), $\alpha$-tocopherol (RR not calculable), or ferritin $(\mathrm{RR}=1.4,95 \% \mathrm{CI}: 0.5,4.4)$ deficiency at delivery, nor was any observed association found between maternal underweight adjBMI during pregnancy and infant zinc $(\mathrm{R} R=0.9,95 \%$ CI: 0.4, 1.9), retinol (RR=0.6, 95\% CI: 0.2, 1.7), $\alpha$-tocopherol $(\mathrm{RR}=0.9,95 \%$ CI: $0.6,1.2)$, ferritin ( $\mathrm{RR}=0.7,95 \%$ CI: $0.4,1.3)$, or $\mathrm{MCHC}$ at birth (RR=2.7, 95\% CI: 0.2, 41.6; Table 4). In addition, maternal zinc, $\alpha$-tocopherol, ferritin, and retinol deficiencies were not associated with age, years of education, the number of persons in the household, gainful employment outside the home, tobacco use during pregnancy, alcohol use during pregnancy, cocaine use during pregnancy, marijuana use during pregnancy, preterm birth, low birth weight or infant CD4 count at birth (data not shown). Women with 
TABLE 3 - Nutritional and inflammatory parameters (as categorical variables) among women (after delivery) and infants (at study visits).

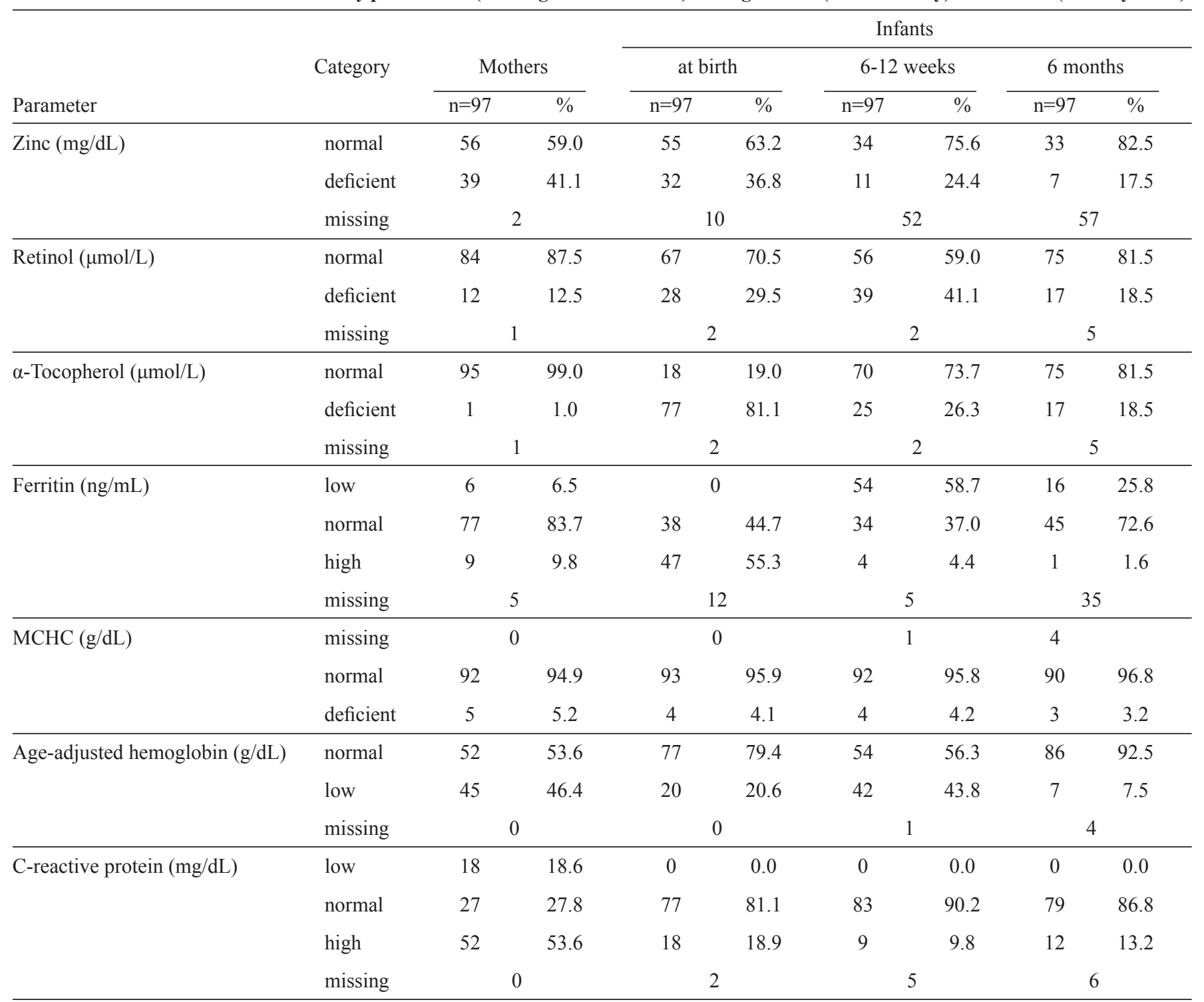

MCHC: mean corpuscular hemoglobin concentration.

acquired immunodeficiency syndrome (AIDS) were less likely to have normal retinol levels than women with asymptomatic or mildly symptomatic HIV disease $(\mathrm{RR}=0.2,95 \% \mathrm{CI}: 0.1,0.6$; data not shown). Low maternal hemoglobin was associated only with low maternal CD4+ T-lymphocyte counts at enrollment. Women who had CD4 cell counts that were $<200$ cells $/ \mathrm{mm}^{3}$ were less likely to have normal hemoglobin levels than were women with CD4+ T-lymphocyte counts that were $\geq 500$ cells $/ \mathrm{mm}^{3}(\mathrm{RR}=0.4$, 95\% CI: 0.3, 0.7; data not shown). Low maternal zinc, ferritin, and hemoglobin were not associated with elevated maternal CRP ( $p$-value $>0.8$ ). No observed associations were found between low maternal levels of zinc $(\mathrm{RR}=1.0,95 \% \mathrm{CI}$ : $0.6,1.7)$, retinol $(\mathrm{RR}=1.5$, 95\%CI: $0.7,3.2$ ), $\alpha$-tocopherol (RR not calculable), or ferritin $(\mathrm{RR}=0.9,95 \% \mathrm{CI}: 0.5,1.6)$ after delivery and corresponding low levels of these micronutrients in the infants at birth ( $p$-value $>0.3$;
Table 5). Additionally, linear regression analysis did not reveal any significant associations between maternal and infant zinc, retinol, $\alpha$-tocopherol, or ferritin levels at birth when the levels were examined on a continuous scale ( $\mathrm{p}$-value $>0.15$ ).

No observed associations were found between low infant zinc, retinol or $\alpha$-tocopherol levels at birth and low levels of the same micronutrients at 6-12 weeks or at six months of age ( $p$-value $>0.1$ ). Low infant ferritin levels at birth were associated with normal infant ferritin levels at 6-12 weeks of age $(p$-value $=0.04)$. This association persisted but was only marginally associated with levels at six months of age (p-value $=0.07)$. Overall, a slightly lower proportion of infants had micronutrient deficiencies at the six-month visit than at birth (data not shown). 
TABLE 4 - Association between maternal adjusted BMI (adjBMI) and micronutrient levels.

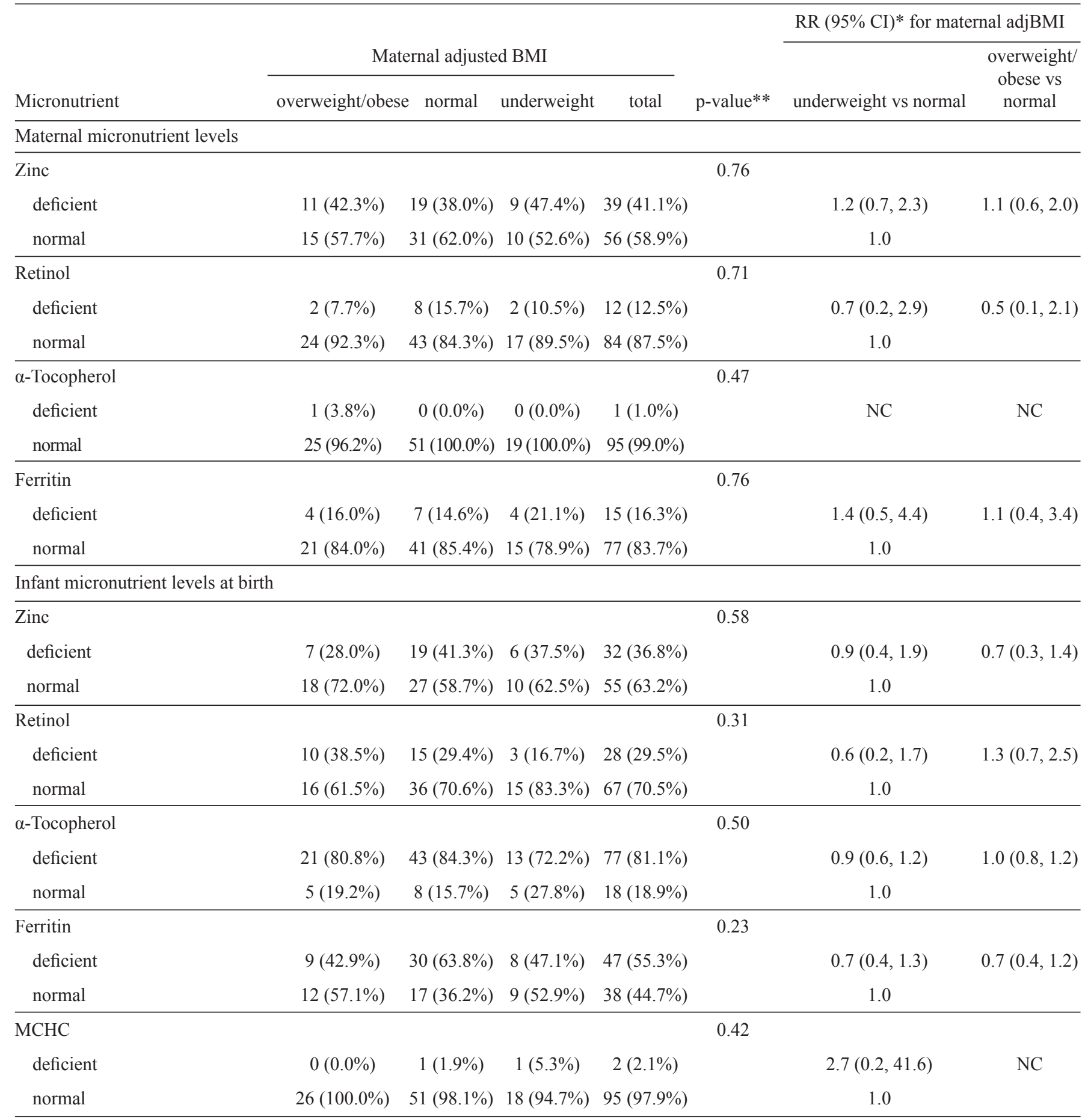

*The relative risks (RRs) and 95\% confidence intervals (CIs) for predicting maternal and infant micronutrient deficiencies as a function of maternal adjBMI were obtained using log binomial regression models.**p-values were obtained using the Fisher-Freeman-Halton test. NC: value could not be calculated; adjBMI: body mass index adjusted for gestational age; MCHC: mean corpuscular hemoglobin concentration. 
TABLE 5 - Association between maternal and infant micronutrient levels.

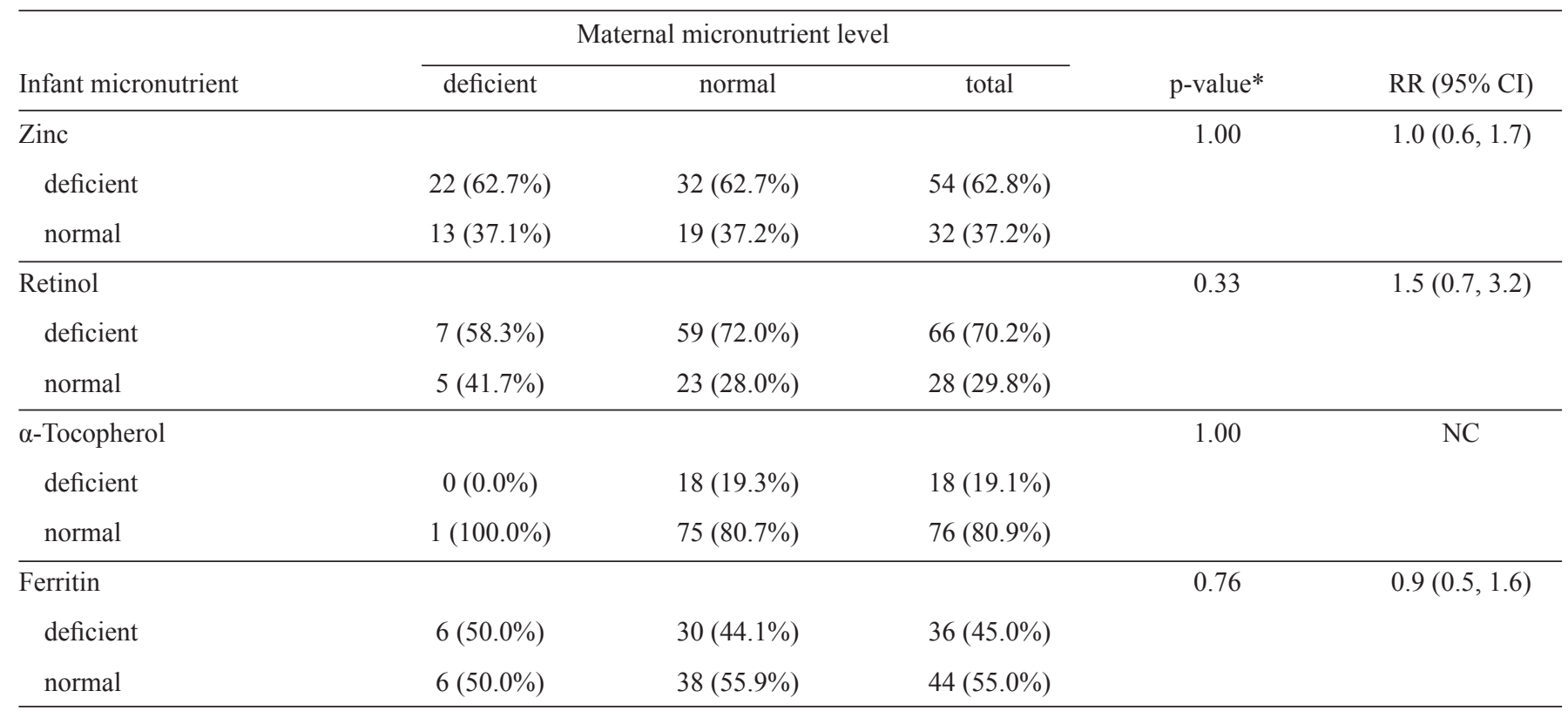

*The p-values were obtained using Fisher's exact test. NC: value could not be calculated. RR: relative risk; CI: Confidence interval.

\section{DISCUSSION}

Micronutrient deficiencies are common in HIV-infected pregnant women and their infants in Brazil. The prevalences of most infant micronutrient deficiencies were lower at six months of age than at birth. Being underweight at the end of pregnancy was not associated with micronutrient deficiency in HIV-infected women or their infants at birth.

We found that $46.4 \%$ of HIV-infected mothers were anemic and that $6.5 \%$ had low levels of ferritin immediately postpartum. Few studies have reported iron deficiency in HIVinfected pregnant women in Latin America; however, some studies have reported an extremely high prevalence of anemia in HIV-infected pregnant women in other countries ${ }^{28} ; 78 \%$ of HIV-infected pregnant women in Burkina Faso were reported to be anemic ${ }^{29}$, and $83 \%$ of HIV-infected pregnant women in Cote d'Ivoire were reported to be anemic ${ }^{30}$.

Factors such as poor nutritional status, HIV infection ${ }^{31}$, ARV therapy ${ }^{32}$, and even vitamin D deficiency ${ }^{33}$ could explain the high prevalence of anemia found in the present sub-study. However, the acute phase response ${ }^{34}$ was not associated with anemia. Few studies have examined lipid-soluble vitamins in HIV-infected pregnant women ${ }^{35-37}$. In Tanzania ${ }^{38}$, approximately 35 and $51 \%$ of HIV-infected pregnant women had low levels of vitamins A and $\mathrm{E}$, respectively. In contrast, only 12.5 and $1 \%$ of women in our sub-study had vitamin A and E deficiencies, respectively, although our cut-off for vitamin E deficiency was lower than that used in Tanzania ${ }^{38}$ (vitamin E level $<9.7 \mu \mathrm{mol} / \mathrm{L}$ ). Zinc deficiency has been reported in children ${ }^{39}$ and in HIV-infected individuals ${ }^{5,40}$ and is thought to be widely prevalent in pregnant women due to hemodilution, decreased albumin levels and insufficient intake ${ }^{41-43}$.
Infants in our sub-study had lower rates of micronutrient deficiencies and higher mean levels of vitamin A and vitamin $\mathrm{E}$ than those found by Monteiro et al. in a group of HIV-infected Latin American children ${ }^{14}$. Nevertheless, $81 \%$ of infants at birth were deficient in $\alpha$-tocopherol, which could partially be explained by increased oxidative stress during delivery. Increased levels of oxidative stress and lipid peroxidation induced by reactive oxygen species (ROS) production play a critical role in the stimulation of HIV replication and immunodeficiency and can be attenuated by the presence of vitamin $\mathrm{E}^{44}$. However, vitamin $\mathrm{E}$ may facilitate HIV entry into cells, and higher plasma vitamin $\mathrm{E}$ levels have been associated with adverse outcomes in HIV-infected individuals ${ }^{45}$. Thus, lower vitamin E levels may constitute a physiological defense mechanism against HIV in infants during delivery.

A high proportion of infants (41\%) had vitamin A deficiency at 6 to 12 weeks, with mean and SD values of 0.8 and $0.4 \mu \mathrm{mol} / \mathrm{L}$, respectively. Chatterjee A et al. also found vitamin A deficiency at six weeks of age; The percentages of infants with plasma vitamin A concentrations less than $0.70 \mathrm{mmol} / \mathrm{L}$ or $0.35 \mathrm{mmol} / \mathrm{L}$ were $89 \%$ and $22 \%$, respectively, in this sub-study ${ }^{46}$. These authors found that higher vitamin A concentrations in plasma at six weeks of age were protective against mortality in children born to HIV-infected women.

Our sub-study had several limitations. Vitamin A deficiency in this sub-study was defined based on WHO recommendations, which are widely accepted and used ${ }^{28}$; however, no consensus regarding a definition of vitamin E deficiency exists. Maternal adjBMI during pregnancy, which is used as a measure of maternal nutritional status, may not actually be a good surrogate for nutritional assessment. Additionally, maternal specimens collected up to seven days postpartum may not accurately 
represent nutritional deficiencies during pregnancy. We did not measure serum lipoprotein and cholesterol, which may be important because serum $\alpha$-tocopherol (vitamin E) concentrations are highly dependent on serum lipid concentrations ${ }^{2,3}$. Likewise, this sub-study did not account for the dietary intake of vitamin $\mathrm{A}$, vitamin $\mathrm{E}$, iron or zinc. All infants were formula-fed and thus received at least some supplementation after birth, although the exact amounts of supplementation are not known. Moreover, the interpretation of serum micronutrient levels is not straightforward due to the wide range of unknown nutrientnutrient and gene-nutrient interactions and physiological and environmental processes involved.

The present sub-study suggests that low serum micronutrient levels are frequently found in HIV-infected pregnant women and their infants and that neither underweight women nor their infants at birth were at increased risk for low serum micronutrient levels. Future studies in different countries that have appropriate designs and that examine genetic and proteomic profiles should be pursued to gain a greater understanding of the interactions among genes, nutrients, and the environment and of micronutrient requirements in HIV-infected and HIVexposed individuals ${ }^{47-50}$.

\section{ACKNOWLEDGMENTS}

We would like to acknowledge all the authors and coauthors, International Site Development Initiative (NISDI) Longitudinal Study in Latin American Countries (LILAC) Study Group and National Institute of Child Health and Human Development (NICHD) for their contribution to this research.

\section{CONFLICT OF INTEREST}

The authors have no competing interests to declare. The findings and conclusions in this report are those of the authors and do not necessarily represent the views of the US National Institutes of Health or the US Department of Health and Human Services.

\section{FINANCIAL SUPPORT}

National Institute of Child Health and Human Development (NICHD) for financial support (Contract \# N01-HD-3-3345, 2002-2007, and Contract \# HHSN267200800001C, Control \# N01-HD-8-0001, 2007-2012).

\section{REFERENCES}

1. Maternal anthropometry and pregnancy outcomes. A WHO Collaborative Study. Bull World Health Organ 1995; 73 (suppl): S1-S98.

2. Black RE. Micronutrients in pregnancy. (Micronutrients, maternal and child health). Br J Nutr 2001; 85 (suppl):S193-S197.
3. Friis H, Gomo E, Kostel P, Ndhlovu P, Nyazema N, Krarup H, et al. HIV and other predictors of serum beta-carotene and retinol in pregnancy: A cross-sectional study in Zimbabwe. Am J Clin Nutr 2001; 73:1058-1065.

4. Lai H, Lai S, Shor-Posner G, Ma F, Trapido E, Baum MK. Plasma zinc, copper, copper:zinc ratio, and survival in a cohort of HIV1-infected homosexual men. J Acquir Immune Defic Syndr 2001; 27:56-62.

5. Jones CY, Tang AM, Forrester JE, Huang J, Hendricks KM, Knox TA, et al. Micronutrient levels and HIV disease status in HIV-infected patients on highly active antiretroviral therapy in the Nutrition for Healthy Living cohort. J Acquir Immune Defic Syndr 2006; 43: 475-482.

6. Wieringa FT, Dijkhuizen MA, Muhilal, Van der Meer JWM. Maternal micronutrient supplementation with zinc and $\beta$-carotene affects morbidity and immune function of infants during the first 6 months of life. Eur J Clin Nutr 2010; 64:1072-1079.

7. Tomkins A. Malnutrition, morbidity and mortality in children and their mothers. Proc Nutr Soc 2000; 59:135-146.

8. Ramalho RA, Flores H, Accioly E, Saunders C. Association between maternal and newborn vitamin A status and economic stratum in Rio de Janeiro, Brazil. Rev Assoc Med Bras 2006; 52:170-175.

9. El Beitune P, Duarte G, Vannucchi H, Quintana SM, FigueiróFilho EA, Morais EN, et al. Serum vitamin A during pregnancy and effects on obstetrics and perinatal outcomes in HIV infected pregnant women. Arch Latinoam Nutr 2004; 54:419-427.

10. Allard JP, Aghdassi E, Chau J, Tam C, Kovacs CM, Salit EM, et al. Effects of vitamin $\mathrm{E}$ and $\mathrm{C}$ supplementation on oxidative stress and viral load in HIV-infected subjects. AIDS 1998; 12:1653-1659.

11. Lee BE, Hong YC, Lee KH, Kim YJ, Kim WK, Chang NS, et al. Influence of maternal serum levels of vitamins $\mathrm{C}$ and $\mathrm{E}$ during the second trimester on birth weight and length. Eur J Clin Nutr 2004; 58:1365-1371.

12. Passi S, Morrone A, Picardo M, De Luca C, Bartoli F, Zurlo A, et al. Blood levels of vitamin E, polyunsaturated fatty acids of phospholipids, lipoperoxides, glutathione peroxidase activity and serological screening for syphilis and HIV in immigrants from developing countries. G Ital Dermatol Venereol 1990; 125: 487-491.

13. Tang J, Faustman C, Hoagland TA, Mancini RA, Seyfert M, Hunt MC. Interactions between mitochondrial lipid oxidation and oxymyoglobin oxidation and the effects of vitamin E. J Agric Food Chem 2005; 53:6073-6079.

14. Monteiro JP, Freimanis-Hance L, Faria LB, Mussi-Pinhata MM, Korelitz J, Vannucchi H, et al. Both human immunodeficiency virusinfected and human immunodeficiency virus-exposed, uninfected children living in Brazil, Argentina, and Mexico have similar rates of low concentrations of retinol, beta-carotene, and vitamin $\mathrm{E}$. Nutr Res 2009; 29:716-722.

15. Berti C, Biesalski HK, Gärtner R, Lapillonne A, Pietrzik K, Poston L, et al. Micronutrients in pregnancy: Current knowledge and unresolved questions. Clin Nutr 2011; 30: 689-701.

16. Levine AM, Berhane K, Masri-Lavine L, Sanchez M, Young M, Augenbraum M, et al. Prevalence and correlates of anemia in a large cohort of HIV-infected women: Women's Interagency HIV Study. J Acquir Immune Defic Syndr 2001; 26:28-35.

17. Read JS, Duarte G, Hance LF, Pinto J, Gouvea MI, Cohen RA, et al. The NICHD International Site Development Initiative perinatal cohorts (2002-09). Int J Epidemiol 2012; 41:642-649.

18. Arnaud J, Fortis I, Blachier S, Kia D, Favier A. Simultaneous determination of retinol, alpha-tocopherol and beta-carotene in serum by isocratic high-performance liquid chromatography. J Chromatogr 1991; 572:103-116. 
19. Ferraz IS, Daneluzzi JC, Vannucchi H, Jordão Jr AA, Ricco RG, Del Ciampo LA, et al. Zinc serum levels and their association with vitamin A deficiency in preschool children. J Pediatr 2007; 83:512-517.

20. Nutrinfo.com - Comunidad Virtual de Profesionales de la Nutrición [Internet]. [Cited 2014 September 27]. Available at: http://www. nutrinfo.com/.

21. Hotz C, Peerson JM, Brown KH. Suggested lower cutoffs of serum zinc concentrations for assessing zinc status: reanalysis of the second National Health and Nutrition Examination Survey data (1976-1980). Am J Clin Nutr 2003; 78:756-764.

22. Yamini S, West KP, Wu L, Dreyfuss ML, Yang DX, Khatry SK. Circulating levels of retinol, tocopherol and carotenoid in Nepali pregnant and postpartum women following long-term beta-carotene and vitamin A supplementation. Eur J Clin Nutr 2001; 55:252-259.

23. Farrell PM, Levine SL, Murphy MD, Adams AJ. Plasma tocopherol levels and tocopherol-lipid relationships in a normal population of children as compared to healthy adults. Am J Clin Nutr 1978; 31:1720-1726.

24. Custer, JW, Rau, RE, Johns Hopkins H. The Harriet Lane handbook: a manual for pediatric house officers. $18^{\text {th }}$ Ed. Philadelphia: Mosby/ Elsevier; 2009.

25. Pearson TA, Mensah GA, Alexander RW, Anderson JL, Cannon RO 3rd, Crigui M, et al. Markers of inflammation and cardiovascular disease: application to clinical and public health practice: A statement for healthcare professionals from the Centers for Disease Control and Prevention and the American Heart Association. Circulation 2003; 107: 499-511.

26. Hatherill M, Tibby SM, Sykes K, Turner C, Murdoch IA. Diagnostic markers of infection: comparison of procalcitonin with $\mathrm{C}$ reactive protein and leucocyte count. Arch Dis Child 1999; 81:417-421.

27. 1993 revised classification system for HIV infection and expanded surveillance case definition for AIDS among adolescents and adults. MMWR Recomm Rep 1992; 41:1-19.

28. World Health Organization (WHO). Global prevalence of vitamin A deficiency in populations at risk 1995-2005. World Heal Organ Glob Database Vitam A Defic 2009; 2009. doi: 9789241598019.

29. Meda N, Dao B, Ouangré A. HIV, maternal anemia and perinatal intervention using zidovudine. DITRAME Study Group (ANRS 049 Clinical Trial). Int J Gynaecol Obstet 1998; 61:65-66.

30. Ramon R, Sawadogo D, Koko FS, Noba V, Likikouët R, Gourvellec $\mathrm{G}$, et al. Haematological characteristics and HIV status of pregnant women in Abidjan, Cote d'Ivoire, 1995-96. Trans R Soc Trop Med Hyg 1999; 93:419-422.

31. Dairo MD, Lawoyin TO, Onadeko MO, Asekun-Olarinmoye EO, Adeniji AO. HIV as an additional risk factors for anaemia in pregnancy: evidence from primary care level in Ibadan, Southwestern Nigeria. Afr J Med Med Sci 2005; 34:275-279.

32. Volberding PA, Baker KR, Levine AM. Human immunodeficiency virus hematology. Hematology Am Soc Hematol Educ Program 2003; 2003:294-313

33. Shin JY, Shim JY. Low vitamin D levels increase anemia risk in Korean women. Clin Chim Acta 2013; 421:177-180.

34. Weiss G, Goodnough LT. Anemia of chronic disease. N Engl J Med 2005; 352:1011-1023.

35. Ahmed F, Azim A, Akhtaruzzaman M. Vitamin A deficiency in poor, urban, lactating women in Bangladesh: factors influencing vitamin A status. Public Health Nutr 2003; 6:447-452.
36. Keverenge-Ettyang GA, van Marken Lichtenbelt W, Esamai F, Saris W. Maternal nutritional status in pastoral versus farming communities of West Pokot, Kenya: differences in iron and vitamin A status and body composition. Food Nutr Bull 2006; 27:228-235.

37. Zvandasara P, Hargrove JW, Ntozini R, Chidawanika H, Mutasa $\mathrm{K}$, Lliff PJ, et al. Mortality and morbidity among postpartum HIVpositive and HIV-negative women in Zimbabwe: risk factors, causes, and impact of single-dose postpartum vitamin A supplementation. J Acquir Immune Defic Syndr 2006; 43:107-116.

38. Mehta S, Spiegelman D, Aboud S, Giovannucci EL, Msanga GI, Hertzmark E, et al. Lipid-soluble vitamins A, D, and E in HIVinfected pregnant women in Tanzania. Eur J Clin Nutr 2010; 64: 808-817.

39. Bhutta ZA, Black RE, Brown KH, Gardner JM, Gore S, Hidayat A, et al. Prevention of diarrhea and pneumonia by zinc supplementation in children in developing countries: pooled analysis of randomized controlled trials. Zinc Investigators' Collaborative Group. J Pediatr 1999; 135:689-697.

40. Caulfield LE, Zavaleta N, Shankar AH, Merialdi M. Potential contribution of maternal zinc supplementation during pregnancy to maternal and child survival. Am J Clin Nutr 1998; 68 (suppl): S499-S508.

41. Wellinghausen N. Immunobiology of gestational zinc deficiency. Br J Nutr 2001; 85(suppl II):81-86.

42. Osendarp SJM, West CE, Black RE. The need for maternal zinc supplementation in developing countries: an unresolved issue. J Nutr 2003; 133:S817-S827.

43. Falcone EL, Mangili A, Tang AM, Jones CY, Woods MN, Polak JF, et al. Micronutrient concentrations and subclinical atherosclerosis in adults with HIV. Am J Clin Nutr 2010; 91:1213-1219.

44. Allard JP, Aghdassi E, Chau J, Salit I, Walmsley S. Oxidative stress and plasma antioxidant micronutrients in humans with HIV infection. Am J Clin Nutr 1998; 67:143-147.

45. Graham SM, Baeten JM, Richardson BA, Bankson DD, Lavreys L, Ndinya-Achola JO, et al. Higher pre-infection vitamin E levels are associated with higher mortality in HIV-1-infected Kenyan women: a prospective study. BMC Infect Dis 2007; 7:63.

46. Chatterjee A, Bosch RJ, Hunter DJ, Manji K, Msamanga GI, Fawzi WW. Vitamin A and vitamin B-12 concentrations in relation to mortality and morbidity among children born to HIV-infected women. J Trop Pediatr 2010; 56:27-35.

47. Killip S, Bennett JM, Chambers MD. Iron deficiency anemia. Am Fam Physician 2007; 75: 671-678.

48. Allen LH, Peerson JM, Olney DK. Provision of multiple rather than two or fewer micronutrients more effectively improves growth and other outcomes in micronutrient-deficient children and adults. J Nutr 2009; 139:1022-1030.

49. Irlam JH, Visser MM, Rollins NN, Siegfried N. Micronutrient supplementation in children and adults with HIV infection. Cochrane Database Syst Rev 2010. CD003650.

50. Fawzi WW, Msamanga GI, Kupka R, Spiegelman D, Villamor E, Mugusi F, et al. Multivitamin supplementation improves hematologic status in HIV-infected women and their children in Tanzania. Am J Clin Nutr 2007; 85:1335-1343. 